e-mail: r-mogutnov@mail.ru

Зеликова Юлия Андреевна - студентка ФГБОУ ВО "Воронежский государственный университет инженерных технологий", г. Воронеж, Российской Федерации,e-mail: Leencha@ya.ru

\title{
Information about authors
}

Kozlov Vyacheslav Gennadievich - Associate Professor of Department of exploitation of transport and technological machines, Federal State Budget Education Institution of Higher Education " Voronezh State Agrarian University named after Emperor Peter the Great", Ph.D. in Engineering, Associate Professor, Voronezh, Russian Federation; e-mail: vya-kozlov@yandex.ru.

Skrypnikov Alexey Vasilyevich - Professor, Department of information security of the "Voronezh state University of engineering technologies", doctor of technical Sciences, Professor, Voronezh, Russian Federation; e-mail: skrypnikovvsafe@mail.ru

Mikova Elena Y.- external information security department FSBEI HE"Voronezh State University of Engineering Technologies", Voronezh, Russian Federation,e-mail: Leencha@ya.ru

Mogutnov Roman Viktorovich - external information security department FSBEI HE "Voronezh State University of Engineering Technologies", Voronezh, Russian Federation,e-mail: r-mogutnov@ mail.ru

Zelikova Yuliya Andreevna.- a student of the "Voronezh State University of Engineering Technologies", Voronezh, Russian Federation,e-mail: Leencha@ya.ru

DOI:10.12737/article_5b2406128d42b4.62955095

УДК 625.863 .4

\section{ПОВЫШЕНИЯ ТЕХНОЛОГИЧЕСКОЙ ЭФФЕКТИВНОСТИ СТРОИТЕЛЬСТВА ЛЕСОВОЗНЫХ АВТОМОБИЛЬНЫХ ДОРОГ ИЗ НИЗКОПРОЧНЫХ КАМЕННЫХ МАТЕРИАЛОВ}

\author{
доктор технических наук, доцент И.Н. Кручинин ${ }^{1}$ \\ Ведущий инженер В.А. Ращектаев
}

1- ФГБОУ ВО «Уральский государственный лесотехнический университет», г. Екатеринбург, Российская Федерация

2- ООО «Проектное бюро Р1», г. Екатеринбург, Российская Федерация

В современных условиях ведения лесопромышленной деятельности для строительства и ремонта лесовозных автомобильных дорог, возможность применения высококачественных кондиционных строительных материалов для устройства конструктивных слоев дорожных одежд становится экономически трудно реализуемой. В то же время использование местных каменных низкопрочных материалов сопряжено с рядом технологических и эксплуатационных трудностей. При отсутствии вяжущего материала основным физико-механическим параметром, оценивающим эксплуатационные характеристики дороги будет являться структурная прочность конструктивного слоя. Нами были проведены лабораторные исследования по изучению изменения зернового состава при уплотнении каменных материалов из различных горных пород. Проведена оценка степени влияния размеров измельченных частиц на структурную прочность слоя покрытия. Изучено влияния крупности частиц на удерживающие силы между ними. Было показано, что частицы крупностью менее 0,25 мм способны образовывать помимо физических связей еще и химические, цементирующие связи за счет реакции с водой. На основе этих данных изучено влияние мелкого заполнителя на величину контактных усилий между частицами различных каменных материалов и на скорость его измельчения. Рассмотрено влияние катков на уплотняемый каменный материал при строительстве лесовозных дорог и получены зависимости его взаимодействия с виброваль- 


\title{
Лесоинжерное дело
}

цами катков. Установлено, что время воздействия вальца, с учетом деформации уплотняемого материала, зависит от периода колебаний вибровозбудителя, времени контакта материала с вальцом, размера пятна контакта и скорости движения катка. Т.е. в процессе измельчения частиц сначала происходит уменьшение давления между зернами каменного материала до предела, когда рост числа контактов замедляется, а затем происходит перераспределение усилий в щебеночном слое. Разработаны рекомендации по уплотнению конструктивных слоев при строительстве дорожных одежд лесовозных автомобильных дорог с использованием местных низкопрочных каменных материалов и проведено сравнение их с кондиционными высокопрочными материалами.

Ключевые слова: лесовозные автомобильные дороги, низкопрочный каменный материал, щебень, зерновой состав, виброкаток, уплотнение.

\section{INCREASING TECHNOLOGICAL EFFICIENCY OF FORESTRY ROADS CONSTRUCTION FROM LOW STRENGTH STONE MATERIALS}

\author{
DSc (Engineering), Associate Professor I.N. Kruchinin ${ }^{1}$ \\ Leading engineer V.A. Rashchektaev ${ }^{2}$
}

\section{1- FSBEI HE "Ural State Forest Engineering University", Yekaterinburg, Russian Federation 2- LLC "Design Bureau P1", Yekaterinburg, Russian Federation}

In the current conditions of conducting forestry activities for the construction and repair of logging roads, the possibility of using high-quality conditional building materials for the construction of structural layers of road clothes becomes economically difficult to realize. At the same time, the use of local stone low-strength materials involves a number of technological and operational difficulties. In the absence of binding material, the main physico-mechanical parameter evaluating the performance characteristics of the road is structural strength of construction layer. We conducted laboratory reserach to study the change in the grain composition during compaction of stone materials from various rocks. The influence degree of size of crushed particles on the structural strength of coating layer has been estimated. The influence of the particle size on the restraining forces between them has been studied. It has been shown that particles smaller than $0.25 \mathrm{~mm}$ are able to form, in addition to physical bonds, chemical and cementing bonds due to the reaction with water. On the basis of these data, the influence of fine aggregate on the magnitude of contact forces between particles of various stone materials and on the rate of its grinding has been studied. The influence of rollers on the compacted stone material during the construction of logging roads is considered and the dependences of its interaction with vibration rolls have been obtained. It is established that the time of action of the roll, taking into account the deformation of the material to be compacted, depends on oscillation period of vibration exciter, time of contact of the material with the roller, the size of the contact spot and the speed of the roller. Those. in the process of grinding the particles, the pressure between the grains of stone material first decreases to the point where the growth in the number of contacts slows down, and then the forces are redistributed in the crushed stone. Recommendations have been developed for compaction of structural layers in the construction of road clothes for forest roads with the use of local low-strength stone materials and their comparison with high-strength conditioned materials has been made.

Keywords: logging roads, low-strength stone material, crushed stone, grain composition, vibration rolls, compaction.

В современной практике строительства, ремонта и реконструкции лесовозных автомобильных дорог основными доступными дорожностроительными материалами служат местные каменные материалы. Они являются либо отходами промышленности, либо низкопрочными каменными материалами, да еще и обладающими непостоянными зерновыми составами $[1,4]$. При производстве работ изменение их физико-механических характеристик становится неизбежным, а отсутствие 
вяжущего материала между частицами требует иного подхода к технологии строительства.

Известно, что основным физикомеханическим параметром таких дискретных сред будет являться их структурная прочность $[3,10]$. В структуре таких сред крупные фракции выполняют роль скелета, то есть пространственного каркаса, выдерживающего основные нагрузки от движущегося лесовозного транспорта. Мелкие частицы в свою очередь выполняют не только роль заполнителя пустот в каркасе, но и при определенном минералогическом составе выполняющие роль слабосвязующего (цементирующего) вещества $[1,4,6]$.

Основной целью данной работы является изучение процессов измельчения и уплотнения низкопрочных каменных материалов при строительстве конструктивных слоев лесовозных автомобильных дорог с применением гладковальцовых виброкатков.

В качестве предмета исследования, в лабораторных условиях были проведены испытания по измельчению каменных материалов. Было рассмотрено изменение зернового состава каждой отдельной фракции низкопрочного каменного материала (известняковый щебень, марка прочности 400) в зависимости от количества циклов приложения внешней нагрузки. С целью сравнительных испытаний параллельно были проведены такие же исследования и на кондиционных каменных материалах (доломитовый щебень, марка прочности 1000). Изменения зернового состава каждой отдельной фракции каменного материала в зависимости от циклов нагружения подробно рассмотрены в наших работах $[3,4]$.

В качестве показателя степени измельчения частиц каменного материала будем использовать количество образующихся при этом мелких частиц: для щебня фракции 10-20 мм это частицы размером $<5$ мм; для фракции 20-40 мм частицы размером $<10$ мм; для фракции 40-70 мм частицы $<20$ мм.

Радиус частиц в процессе измельчения постоянно изменяется. Поэтому, для возможности расчета контактных усилий между частицами, изменение среднего значения радиуса определяем по выражению:

$$
R_{i}=R_{0} \cdot \frac{\left(f_{i}^{0-5}+f_{i}^{5-10}+f_{i}^{10-20}\right)}{100}
$$

где $R_{i}$ - средний радиус частиц на $i$-ом цикле приложения нагрузки, мм;

$R_{0}$ - средний радиус частиц до уплотнения, мм;

$f_{i}^{0-5}$ - процент фракции 0-5 мм на $i$-ом цикле приложения нагрузки;

$f_{i}{ }^{5-10}$ - процент фракции 5-10 мм на $i$-ом цикле приложения нагрузки;

$f_{i}^{10-20}$ - процент фракции 10-20 мм на $i$-ом цикле приложения нагрузки.

Как показано в работах [2,8,11], именно размер этих частиц оказывает непосредственное влияние на сдерживающие силы между частицами зернистых материалов и влияет на технологическую эффективность уплотняющих машин при строительстве.

На рисунке 1 и в таблице 1 представлены кривые зернового состава малопрочного известнякового щебня марки 400 в зависимости от количества приложений нагрузки, полученные в лабораторных условиях.

Полученные данные были использованы в математической модели уплотнения каменных материалов при строительстве конструктивных слоев лесовозных автомобильных дорог. В основу математического описания было положено определение удерживающих сил между частицами [3]. В связи с этим, при расчете удерживающей силы между частицами приняты следующие допущения: рассматриваем только бинарную структуру смеси (два типоразмера частиц); в модели рассматриваем только силы трения между частицами; эквивалентные частицы каменной пыли равномерно распределены по всему объему образованной при уплотнении смеси; средний радиус частиц принят размером $<0,25$ мм [7].

Для расчета контактных усилий возникающих между частицами низкопрочных каменных материалов, с учетом принятых допущений средний расчетный радиус частицы $R_{c}$ определяется по формуле 


$$
R_{c}=R_{0} \cdot\left(1-k_{i}\right)+\frac{R_{i} a_{i}}{100},
$$

где $R_{0}$ - средний радиус частиц до уплотнения, мм; $R_{i}$ - средний радиус частиц на $i$-ом цикле приложения нагрузки, мм;

$k_{i}$ - степень измельчения;

$a_{i}$-параметр учета цементации,

$$
a_{i}=\frac{f_{i}^{0-0,25}}{100} ;
$$

$f_{i}^{0-0,25}$ - содержание щебня фракции $<0,25$ мм, $\%$.

В результате лабораторных исследований было установлено, что известняковый щебень прочности 400 гораздо сильнее подвержен измельчению при воздействиях многократных циклических нагрузок, чем прочный доломитовый щебень. При этом увеличение сдерживающих сил между частицами за счет цементации у слабых каменных материалов может составлять от 5 до 30 процентов.

В тоже время необходимо учитывать, что основной технологической операцией при строительстве лесовозных автомобильных дорог было и остается уплотнение дорожными вибрационными катками. Современный парк этих дорожно-строительных машин для уплотнения дорожно-строительных материалов практически полностью покрывает потребности в производстве работ. Однако строительство лесовозных автомобильных дорог имеет свои особенности.

Как показано в работах $[2,11,12]$ степень уплотнения конструктивных слоев зависит не только от скорости движения, числа проходов, но и частоты и вынуждающей силы вибратора. Таким образом, при уплотнении виброкатком на уплотняемую поверхность действует обобщающая сила, состоящая из статической, от силы веса катка и динамической. При этом время импульса, с учетом деформации уплотняемого материала, зависит от периода колебаний вибровозбудителя, а время контакта материала с вальцом от размеров пятна контакта и скорости движения катка (таблица 2).

Для расчетной модели было принято: количество проходов катка - не менее 12. Первые три прохода и последний на скорости движения катка равной от 1,5 до 2 км/ч, на остальных проходах 7,5 км/ч. При этом пятно контакта вальца катка с материалом принимало значение от 0,05 м (начальная стадии уплотнения), до 0,01 м на последних проходах катка.

Оказалось, что степень воздействия на материал за цикл нагрузки уменьшается, несмотря на то, что нормальное давление под вальцом увеличивается, а набор плотности конструкции из низкопрочного каменного материала сопровождается упаковкой и перемещением его частиц. В процессе измельчения частиц происходит уменьшение давления между зернами каменного материала до предела, когда рост числа контактов замедляется и происходит перераспределение усилий в щебеночном слое. Последующее уплотнение приводит к росту сдерживающих усилий между зернами, вплоть до достижения оптимального гранулометрического состава и нормативного значения межзерновой пустотности (от 12 до 14 \%). На рисунке 2 представлено изменение межзерновой пустотности в зависимости от количества приложений нагрузки для низкопрочного известнякового щебня марки 400. При этом скорость его измельчения уменьшатся только после количества циклов приложения нагружений, эквивалентных двадцати проходов виброкатка по одному следу. Сравнительный расчет контактных усилий, возникающих между частицами каменного материала с учетом образования химически активной каменной пыли и конгломератных образований показал, что исследуемый известняковый щебень гораздо сильнее подвержен измельчению при воздействиях многократных циклических нагрузок, чем прочный доломитовый [5]. Разработанные модели и проведенный комплекс лабораторных исследований позволил заново проанализировать изменение состояния конструктивных слоев лесовозных автомобильных дорог при воздействии на них уплотняющей нагрузки. 
В процессе уплотнения каменный материал конструктивного слоя проходит следующие фазо-

вые состояния (рис.3).

Зерновой состав после испытания в лабораторной установке известнякового щебня марки 400

\begin{tabular}{|c|c|c|c|c|c|}
\hline \multirow{2}{*}{$\begin{array}{c}\text { Количество приложений } \\
\text { нагрузки }\end{array}$} & \multicolumn{5}{|c|}{ Количество частиц меньше фракции, \% } \\
\cline { 2 - 6 } & 40 & 20 & 10 & 5 & 0 \\
\hline 3 & 100 & 39,5 & 20,4 & 12,6 & 0 \\
\hline 5 & 100 & 44,1 & 23,7 & 15,3 & 0 \\
\hline 7 & 100 & 49,4 & 27,5 & 21,2 & 0 \\
\hline 10 & 100 & 56,3 & 31,4 & 25,3 & 0 \\
\hline 15 & 100 & 61,2 & 37,3 & 28,1 & 0 \\
\hline
\end{tabular}

Таблица 2

Степень влияния уплотняющей нагрузки в зависимости от числа проходов катка, с учетом площадки контакта вальца с материалом

\begin{tabular}{|c|c|c|c|c|c|c|c|}
\hline $\begin{array}{l}\text { Количество проходов } \\
\text { катка по одному следу }\end{array}$ & 2 & 4 & 6 & 8 & 10 & 12 & 14 \\
\hline $\begin{array}{l}\text { Статическая нагрузка на } \\
\text { валец, МПа }\end{array}$ & 3,46 & 3,46 & 3,46 & 3,46 & 3,46 & 3,46 & 3,46 \\
\hline $\begin{array}{l}\text { Вынуждающая сила } P_{\text {дин }}, \\
\text { кН }\end{array}$ & 207 & 207 & 207 & 207 & 207 & 207 & 207 \\
\hline Ширина вальца, м & 2,07 & 2,07 & 2,07 & 2,07 & 2,07 & 2,07 & 2,07 \\
\hline $\begin{array}{l}\text { Динамическая нагрузка, } \\
\text { МПа }\end{array}$ & 2,19 & 2,72 & 3,60 & 5,29 & 6,92 & 10,00 & 12,30 \\
\hline Скорость укатки, км/ч & 1,75 & 2,79 & 4,36 & 5,93 & 6,71 & 7,5 & 7,5 \\
\hline Пятно контакта $S_{\text {кон }}$, м & 0,45 & 0,36 & 0,27 & 0,18 & 0,14 & 0,10 & 0,10 \\
\hline $\begin{array}{l}\text { Время воздействия на } \\
\text { материал } t_{\kappa о н} \text {, с }\end{array}$ & 0,094 & 0,047 & 0,023 & 0,011 & 0,008 & 0,005 & 0,005 \\
\hline $\begin{array}{l}\text { Количество циклов } \\
\text { нагружения, } n_{u}\end{array}$ & 2,8 & 1,4 & 0,7 & 0,3 & 0,2 & 0,14 & 0,14 \\
\hline $\begin{array}{l}\text { Индекс интенсивности } \\
\text { нагружения } N\end{array}$ & 61,7 & 38,8 & 24,8 & 18,2 & 16,1 & 14,4 & 12,5 \\
\hline
\end{tabular}




\section{Лесоинжерное дело}

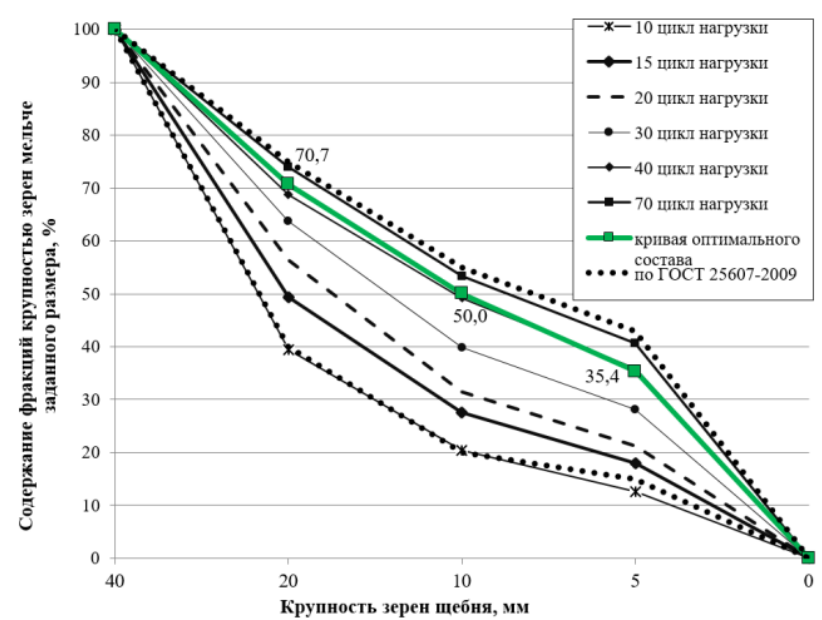

Рис. 1.Кривые зернового состава низкопрочного известнякового щебня марки 400 в зависимости от количества приложений нагрузки

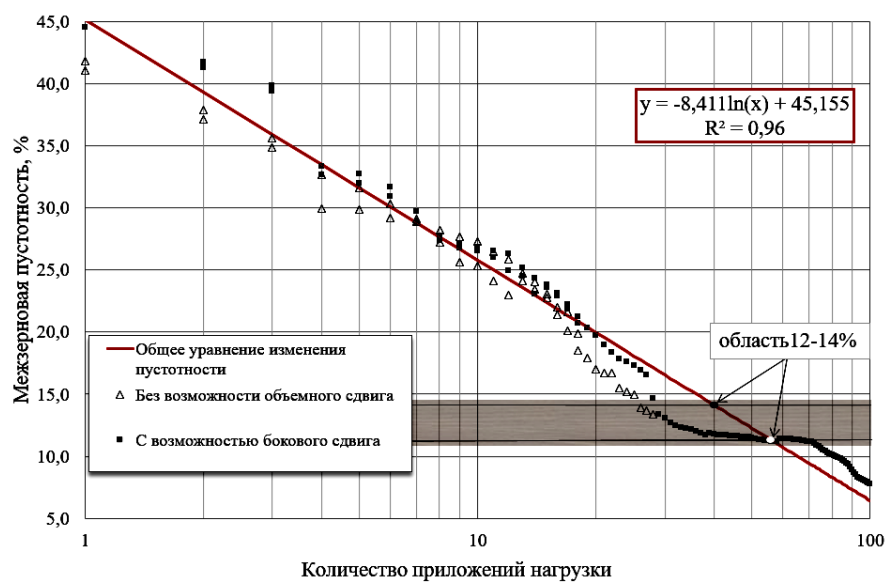

Рис.2. Изменение межзерновой пустотности в зависимости от количества приложений нагрузки для низкопрочного известнякового щебня марки 400

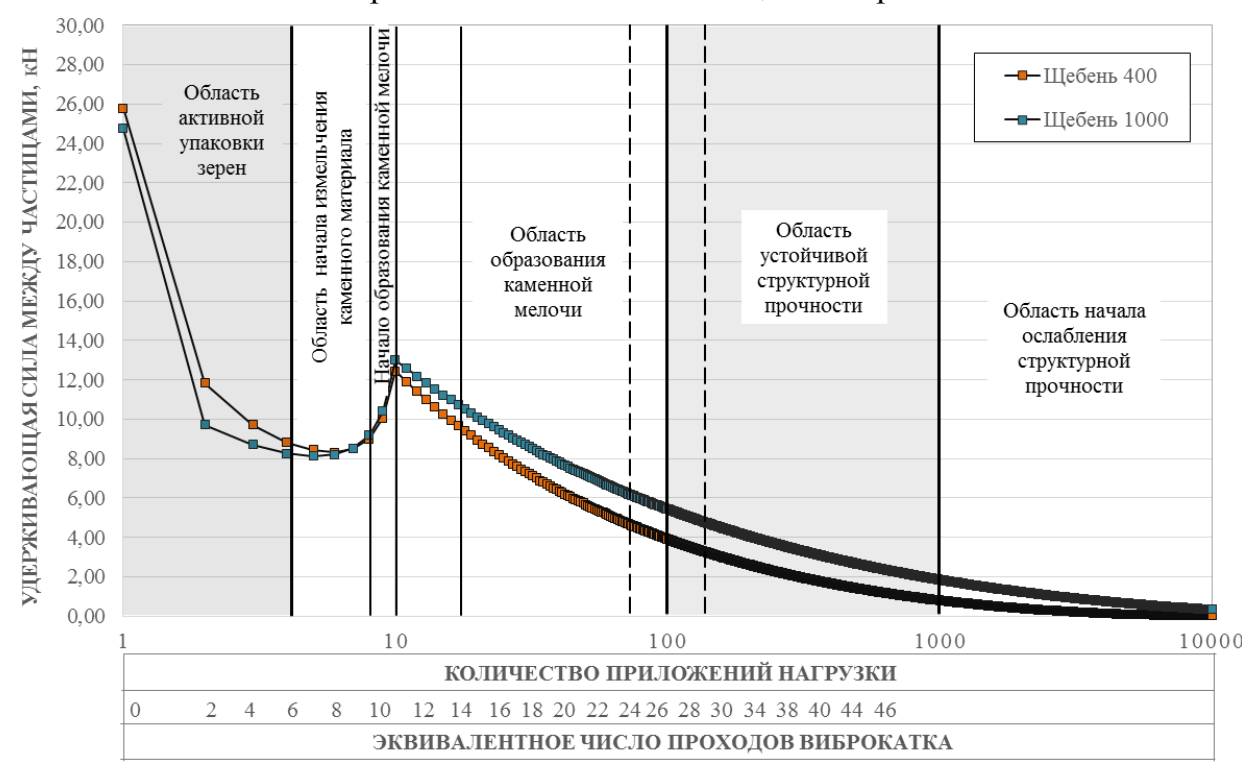

Рис.3. Изменения физико-механических характеристик конструктивного слоя лесовозных дорог в процессе строительства 
Область активной упаковки зерен каменного материала, характеризующейся активным перемещением относительно друг друга зерен материала. Область начального измельчения каменного материала, со сломом частиц и созданием прочного каркаса. Область образования каменной мелочи, с заполнением межзернового пространства и перераспределением усилий между частицами. Область образования устойчивой структурной прочности конструктивного слоя, с последующим измельчением частиц. Область ослабления структурной прочности в результате образования повышенного содержания мелкого заполнителя, сопровождающийся потерей прочности дорожного покрытия.

Вывод: Разработанная методика строительства конструктивных слоев лесовозных авто- мобильных дорог, с учетом взаимодействия виброкатка с уплотняющим материалом, позволила прогнозировать изменения их физико-механических характеристик. Показано, что для достижений нормативных показателей, для покрытий из низкопрочных материалов необходимо приложить от 50и до 70-и циклов внешнего нагружения, что эквивалентно от 10-и до 12-и проходов виброкатка по одному следу. В случае увеличения количества проходов виброкатка от воздействия уплотняющей техники происходит его дальнейшее измельчение с последующей потерей структурной прочности слоя. В случае использования кондиционных высокопрочных каменных материалов уплотнение следует прекращать при достижении 16-и проходов виброкатка по следу.

\section{Библиографический список}

1. Инновационные технологии проектирования и строительства автомобильных дорог: монография / Д. Г. Неволин, В. Н. Дмитриев, Е.В. Кошкаров и др. ; под ред. Д. Г. Неволина, В. Н. Дмитриева; Авторский коллектив: Д. Г. Неволин, В. Н. Дмитриев, Е.В. Кошкаров, С.А. Чудинов, Н.А. Гриневич, И.Н. Кручинин, С.Г. Кривошеев, А.С. Скрипкин, В.Е. Кошкаров, Е.Н. Шаламова, В.Л. Бутенко. - Екатеринбург : УрГУПС, $2015 .-291$ с.

2. Кручинин, И.Н. Обоснование технологических параметров уплотняющей техники при строительстве лесовозных автомобильных дорог / И.Н. Кручинин, С.И. Сушков // Строительные и дорожные машины: научнотехнический и производственный журнал. - 2016. - №5.- С.32-35

3. Ращектаев В.А., Кручинин И.Н. Моделирование процесса уплотнения оснований дорожных одежд лесовозных автомобильных дорог из слабых материалов / Известия Коми научного центра Уральского отделения Российской академии наук: научный журнал. - Сыктывкар, 2014. - Вып. 2(18). -С.80-82.

4. Ращектаев В.А., Чудинов С.А. Исследование свойств щебеночно-песчаных смесей металлургических шлаков для устройства монолитных оснований дорожных одежд // Современные проблемы науки и образования: научный журнал. - 2014. - № 4; URL: www.science-education.ru/118-14056 (дата обращения: 11.08.2014).

5. Свидетельство о государственной регистрации программы для ЭВМ №2014615551. Российская Федерация. Программа для расчета изменения физико-механических характеристик легкоуплотняемых каменных материалов «Compaction of crushed rocks» / В.А. Ращектаев, И.Н. Кручинин; заявитель и правообладатель ФГБОУ ВПО Уральский государственный лесотехнический университет. - №2014613274; заявл. 11.04.2014; зарегистрировано 28.05.2014.

6. Hagerty, M. M., Hite, D. R., Ullrich, C. R., \& Hag, Onedimensional high-pressure compression of granular media // Journal of Geotechnical Engineering, т. 1, № 119, pp. 1-18, 1993.

7. Nakata, Y., Hyodo, M., Hyde, Microscopic particle crushing of sand subjected to high pressure onedimensional compression // Soils and Foundations, т. 1, № 41, pp. 69-82., 2001.

8. Özgan, E. et al. Multi-faceted investigation and modeling of compaction parameters for road construction / E. Özgan, S. Serin, İ. Vural // Journal of Terramechanics, August . -2015. - Volume 41. - Issue 4./ Electronic recourse/ - mode access: http://dx.doi.org/10.1016/ j.jterra.2015.02.005. 
9. Siripun, K. et.al. Mechanical Behavior of Unbound Granular Road Base Materials under Repeated Cyclic Loads / K. Siripun, H. Nikraz, P. Jitsangiam //International Journal of Pavement Research and Technology, Jan. -2011. - Vol.4. - No.1. - P. 56-66.

10. Sweere, G.T.H. Unbound granular bases for roads [Text] / PhD Dissertation, Delft University of Technology, the Netherlands. $-1990 .-127$ p.

11. Thom, N.H. and Brown S.F. The effect of grading and density on the mechanical properties of a crushed dolomitic limestone / N.H. Thom, S.F. Brown// Proceedings / 14th ARRB Conference, 28 August - 2 September 1988 Canberra, Australian Capital Territory. -1988. - P. $94-100$.

12. Wolff, H. and Visser, A.T., Incorporating elasto-plasticity in granular layer pavement design [Text] / H. Wolff, A.T. Visser // Transportation Engineering. - 1994. -Vol. 105. - P. 259-272.

\section{Reeferences}

1. Innovatsionnye tekhnologii proektirovaniya i stroitel'stva avtomobil'nykh dorog: monografiya [Innovative technologies for the design and construction of highways: monograph] / D.G. Nevolin, V.N. Dmitriev, E.V. Koshkarov and others; Ed. D.G. Nevolina, V.N. Dmitrieva; Authors: D. G. Nevolin, V. N. Dmitriev, E.V. Koshkarov, S.A. Chudinov, N.A. Grinevich, I.N. Kruchinin, S.G. Krivosheev, A.S. Skripkin, V.E. Koshkarov, E.N. Shalamova, V.L. Butenko. - Ekaterinburg: USURT, 2015. - 291 c.

2. Kruchinin I. N. Sushkov S. I. Obosnovanie tekhnologicheskikh parametrov uplotnyayushchey tekhniki pri stroitel'stve lesovoznykh avtomobil'nykh dorog [Substantiation of technological parameters of sealing equipment in the construction of logging roads]. Stroitel'nye i dorozhnye mashiny: nauchno-tekhnicheskiy i proizvodstvennyy zhurnal [Construction and road machines: scientific and technical and production magazine]. - 2016. - №5. P.32-35.

3. Rashchektaev V.A., Kruchinin I.N. Modelirovanie protsessa uplotneniya osnovaniy dorozhnykh odezhd lesovoznykh avtomobil'nykh dorog iz slabykh materialov [Modeling process seal bases of road clothes of logging roads from weak materials]. Izvestiya Komi nauchnogo tsentra Ural'skogo otdeleniya Rossiyskoy akademii nauk: nauchnyy zhurnal [Proceedings of the Komi Science Center, Ural Branch of the Russian Academy of Sciences: scientific journal]. - Syktyvkar, 2014. - Vol. 2 (18). -S.80-82.

4. Rashchektaev V.A., Chudinov S.A. Issledovanie svoystv shchebenochno-peschanykh smesey metallurgicheskikh shlakov dlya ustroystva monolitnykh osnovaniy dorozhnykh odezhd [Studying the properties of rubble-sand mixtures metallurgical slag for the device monolithic bases of road clothes]. Sovremennye problemy nauki i obrazovaniya: nauchnyy zhurnal [Modern-recurrent problems of science and education: a scientific journal]. - 2014. № 4; URL: www.science-education.ru/118-14056 (reference date: 11.08.2014).

5. Svidetel'stvo o gosudarstvennoy registratsii programmy dlya EVM №2014615551. Rossiyskaya Federatsiya. Programma dlya rascheta izmeneniya fiziko-mekhanicheskikh kharakteri-stik legkouplotnyaemykh kamennykh materialov «Compaction of crushed rocks» [Certificate of state registration of the computer №2014615551. Russian Federation]. The program for the calculation of changes in physical and mechanical nature-stick legkouplotnyaemyh stone materials «Cmpaction of crushed rocks» / VA Raschekta-s, IN Kruchinin; applicant and rights holder VPO Ural State Forestry University. - №2014613274; appl. 04/11/2014; registered 05.28.2014.

6. Hagerty, M. M., Hite, D. R., Ullrich, C. R., \& Hag, Onedimensional high-pressure compression of granular media // Journal of Geotechnical Engineering, т. 1, № 119, pp. 1-18, 1993.

7. Nakata, Y., Hyodo, M., Hyde, Microscopic particle crushing of sand subjected to high pressure onedimensional compression // Soils and Foundations, т. 1, № 41, pp. 69-82., 2001.

8. Özgan, E. et al. Multi-faceted investigation and modeling of compaction parameters for road construction / E. Özgan, S. Serin, İ. Vural // Journal of Terramechanics, August . -2015. - Volume 41. - Issue 4./ Electronic recourse/mode access: http://dx.doi.org/10.1016/ j.jterra.2015.02.005. 
9. Siripun, K. et.al. Mechanical Behavior of Unbound Granular Road Base Materials under Repeated Cyclic Loads [Text] / K. Siripun, H. Nikraz, P. Jitsangiam //International Journal of Pavement Research and Technology, Jan. -2011. - Vol.4. - No.1. - P. 56-66.

10. Sweere, G.T.H. Unbound granular bases for roads [Text] / PhD Dissertation, Delft University of Technology, the Netherlands. - 1990. -127 p.

11. Thom, N.H. and Brown S.F. The effect of grading and density on the mechanical properties of a crushed dolomitic limestone [Text] / N.H. Thom, S.F. Brown// Proceedings / 14th ARRB Conference, 28 August - 2 September 1988 Canberra, Australian Capital Territory. -1988. - P. $94-100$.

12. Wolff, H. and Visser, A.T., Incorporating elasto-plasticity in granular layer pavement design [Text] / H. Wolff, A.T. Visser // Transportation Engineering. - 1994. -Vol. 105. - P. 259-272.

\title{
Сведения об авторах
}

Кручинин Игорь Николаевич - профессор кафедры транспорта и дорожного строительства, федеральное бюджетное образовательное учреждение высшего образования «Уральский государственный лесотехнический университет», доктор технических наук, доцент, г. Екатеринбург, Российская Федерация, e-mail: kinaa.k@ уa.ru.

Ращектаев Владимир Александрович - соискатель кафедры транспорта и дорожного строительства, федеральное бюджетное образовательное учреждение высшего образования «Уральский государственный лесотехнический университет», ведущий инженер ООО «Проектное бюро Р1», г. Екатеринбург, Российская Федерация, e-mail: rrash@mail.ru

\section{Information about the authors}

Kruchinin Igor Nikolaevich - Professor of transport and road construction, the federal budget institution of higher education «Ural State Forestry Engineering University», doctor of technical Sciences, Associate Professor, Ekaterinburg, Russia, e-mail: kinaa.k@ya.ru.

Raschektaev Vladimir Alexandrovich - applicant for the faculty position of transport and road construction, the federal budget institution of higher education «Ural State Forestry Engineering University», Lead Engineer LLC "Project Office P1", Ekaterinburg, Russia, e-mail: rrash@mail.ru

DOI: $10.12737 /$ article_5b2406175e7765.44768086

УДК 625.7

\section{ПЛАНИРОВАНИЕ СРОКОВ ЭКСПЛУАТАЦИИ ЗИМНИХ ЛЕСОВОЗНЫХ ДОРОГ НА ОСНОВЕ АНАЛИЗА СТАТИСТИКИ КЛИМАТИЧЕСКИХ ДАННЫХ}

\author{
кандидат технических наук, доцент А.П. Мохирев ${ }^{1}$ \\ кандидат сельскохозяйственных наук, доцент Е.В. Горяева $\mathbf{2}^{2,3}$ \\ магистрант М.П. Мохирев \\ магистрант А.В. Ившина ${ }^{1}$
}

1 - ФГБОУ ВО «Сибирский государственный университет науки и технологий имени академика

М.Ф. Решетнева», филиал СибГУ им. М.Ф. Решетнева в г. Лесосибирске, Лесосибирск, Российская Федерация

2 - ФГАОУ ВО «Сибирский федеральный университет», Красноярск, Российская Федерация, 3 - Институт леса им. В.Н.Сукачева СО РАН, Федеральный исследовательский центр «Красноярский научный центр СО РАН», Красноярск, Российская Федерация

Лесозаготовительная промышленность напрямую зависит от климатических условий. Лесозаготовки ведутся на огромных территориях, занимающих почти половину территории страны. Климатические условия, 